\title{
THE INTERSECTION OF BIVARIATE ORTHOGONAL POLYNOMIALS ON TRIANGLE PATCHES
}

\author{
TOM H. KOORNWINDER AND STEFAN A. SAUTER
}

\begin{abstract}
In this paper, the intersection of bivariate orthogonal polynomials on triangle patches will be investigated. The result is interesting on its own but also has important applications in the theory of a posteriori error estimation for finite element discretizations with $p$-refinement, i.e., if the local polynomial degree of the test and trial functions is increased to improve the accuracy. A triangle patch is a set of disjoint open triangles whose closed union covers a neighborhood of the common triangle vertex. On each triangle we consider the space of orthogonal polynomials of degree $n$ with respect to the weight function which is the product of the barycentric coordinates. We show that the intersection of these polynomial spaces is the null space. The analysis requires the derivation of subtle representations of orthogonal polynomials on triangles. Up to four triangles have to be considered to identify that the intersection is trivial.
\end{abstract}

\section{INTRODUCTION}

In this paper, we will investigate the intersection of bivariate orthogonal polynomials on triangle patches. This problem arises in the theory of a posteriori error estimation for finite element discretizations of elliptic partial differential equations; in particular, if the local polynomial degree of the finite element spaces is increased during the solution process. Before we give the precise mathematical formulation of this problem we will sketch its application in the finite element analysis.

A posteriori error estimation and adaptivity are well established methodologies for the numerical solution of partial differential equations by finite elements (cf. [2], [3], 21, 1], 4], 18], 9], 16], 19], 7]).

Some types of error estimators, for example hierarchical ones (see, e.g., [5], 8], [6]), require explicitly or implicitly the saturation assumption which states that the error on the refined mesh and/or with higher polynomial degree is strictly smaller than the error on the previous mesh/polynomial degree. In the pioneering paper [10] the saturation assumption is proved for the Poisson problem in two spatial dimensions under the assumption that the data oscillations are small. In [16] the convergence of adaptive finite element methods (AFEM) for general (nonsymmetric) second order linear elliptic partial differential equations is proved, where the term "adaptivity" is understood in the sense of adaptive mesh refinement and the polynomial degree stays fixed. The theory in [16 also generalizes the proof of the saturation property to quite general second order elliptic problems and estimates the error on the refined mesh by the error of the coarser mesh plus a data oscillation term.

Received by the editor September 23, 2013 and, in revised form, November 1, 2013.

2010 Mathematics Subject Classification. Primary 65N50, 33C50; Secondary 65N15, 65N30, $33 \mathrm{C} 45$. 


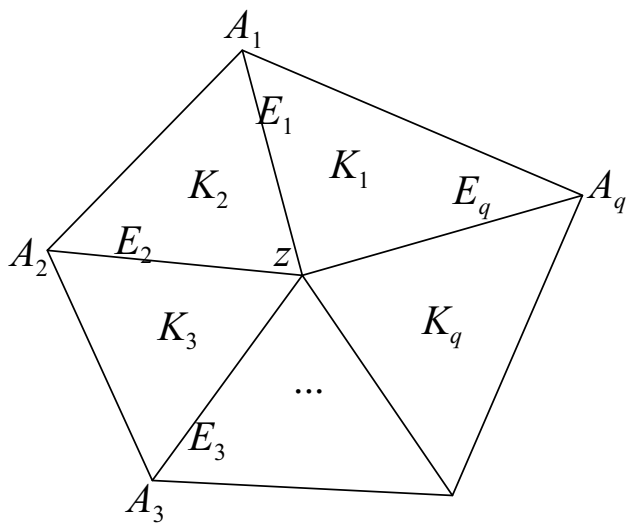

Figure 1. Triangle patch $\mathcal{T}:=\left\{K_{i}: 1 \leq i \leq q\right\}$ around a point $z$. The triangles $K_{i}$ and $K_{i+1}$ share an edge $E_{i}$. Each triangle $K_{i}$ has $z$ as a vertex while its other vertices are denoted by $A_{i-1}$ and $A_{i}$.

For the proof of the saturation assumption for $p$-refinement, i.e., when the local polynomial degree of the finite element space is increased instead of the mesh being refined, a difficulty arises which is related to a polynomial projection property on triangle patches. Here orthogonal polynomials in two variables on a triangle (see [17, 15, 12] ) enter, and the problem just raised is also interesting on its own in that area. By the way, these orthogonal polynomials also have important applications in the field of spectral methods for discretizing partial differential equations and we refer to 13 for further details. In particular, orthogonal polynomials on triangles can be efficiently used for discontinous Galerkin $(\mathrm{dG})$ methods or to discretize boundary integral equation of negative order since no continuity is required across simplex boundaries.

Let us now briefly state the problem which we will solve in this paper. For a two-dimensional domain $D \subset \mathbb{R}^{2}$, the set of bivariate polynomials of maximal total degree $n$ is denoted by $\mathbb{P}_{n}(D)$. Put $\mathbb{P}_{-1}(D):=\{0\}$. Let $z \in \mathbb{R}^{2}$ and let $\mathcal{T}:=\left\{K_{i}: 1 \leq i \leq q\right\}$ denote a triangle patch around $z$, i.e., $\mathcal{T}$ is a set of (open) triangles (cf. Figure 1) which

- are pairwise disjoint,

- have $z$ as a common vertex.

- For all $1 \leq i \leq q$, the triangles $11 K_{i}$ and $K_{i+1}$ have one common edge, denoted by $E_{i}$, which connects the common vertices $z$ and $A_{i}$ of $K_{i}$ and $K_{i+1}$.

Thus

$$
K_{i}=\operatorname{conv}^{\mathrm{o}}\left(z, A_{i-1}, A_{i}\right),
$$

where conv denotes the convex hull of the given points and $\operatorname{conv}^{\mathrm{o}}$ the open interior of this convex hull.

\footnotetext{
${ }^{1}$ We use here the convention $K_{0}:=K_{q}$ and $K_{q+1}:=K_{1}$ and analogously for the vertices $A_{i}$ and the edges $E_{i}$. Clearly $q \geq 3$ holds.
} 
Let $\Omega:=\operatorname{int}\left(\bigcup_{i=1}^{q} \overline{K_{i}}\right)$ and let $\mathfrak{S}:=\Omega \cap\left(\bigcup_{i=1}^{q} \partial K_{i}\right)$ denote the inner mesh skeleton. We denote by $\mathbb{P}_{n}(\mathcal{T})$ the space of piecewise polynomials, i.e.,

$$
\mathbb{P}_{n}(\mathcal{T}):=\left\{f: \Omega \backslash \mathfrak{S} \rightarrow \mathbb{R}|\forall i \in\{1, \ldots, q\} \quad f|_{K_{i}} \in \mathbb{P}_{n}\left(K_{i}\right)\right\} .
$$

We consider $\mathbb{P}_{n}(\Omega)$ as a linear subspace of $\mathbb{P}_{n}(\mathcal{T})$ by its natural embedding.

For $i=1, \ldots, q$ define a weight function $\omega_{i}:=a_{i} b_{i} c_{i}$ on $K_{i}$, where $a_{i}, b_{i}, c_{i}$ are affine linear functions which vanish on the respective edges of $K_{i}$. Thus $\omega_{i}$ is the product of the barycentric coordinates in $K_{i}$ or, in other terms, a cubic bubble function which is positive on $K_{i}$. We define the inner product $(\cdot, \cdot)_{\mathcal{T}}$ on $\mathcal{T}$ by

$$
(u, v)_{\mathcal{T}}:=\sum_{i=1}^{q}(u, v)_{K_{i}}
$$

where

$$
(u, v)_{K_{i}}:=\int_{K_{i}} u(x, y) v(x, y) \omega_{i}(x, y) d x d y .
$$

Denote by $\mathbb{P}_{n-1}^{\perp}\left(K_{i}\right)$ the orthoplement of $\mathbb{P}_{n-1}\left(K_{i}\right)$ in $\mathbb{P}_{n}\left(K_{i}\right)$ with respect to the inner product (1.3). Let $\Pi_{n}^{\mathcal{T}}: \mathbb{P}_{n}(\Omega) \rightarrow \mathbb{P}_{n-1}(\mathcal{T})$ denote the restriction to $\mathbb{P}_{n}(\Omega)$ of the orthogonal projection of $\mathbb{P}_{n}(\mathcal{T})$ onto $\mathbb{P}_{n-1}(\mathcal{T})$ with respect to the inner product (1.2).

Theorem 1.1. Let $n \geq 1$. Then the following three statements are equivalent and each of them holds.

(a) If $u \in \mathbb{P}_{n}(\Omega)$ and $(u, w)_{\mathcal{T}}=0$ for all $w \in \mathbb{P}_{n-1}(\mathcal{T})$ then $u=0$.

(b) $\bigcap_{i=1, \ldots, q} \mathbb{P}_{n-1}^{\perp}\left(K_{i}\right)=\{0\}$.

(c) The map $\Pi_{n}^{\mathcal{T}}: \mathbb{P}_{n}(\Omega) \rightarrow \mathbb{P}_{n-1}(\mathcal{T})$ is injective.

The equivalence of the three statements is trivial, so we can pick one of them as what we aim to prove. It turns out that (b) is the most convenient statement for a proof. Then it is natural to examine first the intersection of two such spaces for adjacent triangles, i.e., $\mathbb{P}_{n-1}^{\perp}\left(K_{i}\right) \cap \mathbb{P}_{n-1}^{\perp}\left(K_{i-1}\right)$. This will be the subject of Section 3. where explicit knowledge of orthogonal polynomials on the triangle for the inner product (1.3), to be summarized in Section 2, is crucial.

By Section 3 the intersection for two adjacent triangles is mostly $\{0\}$, but there are exceptional cases. For these cases it is necessary to consider the intersection of spaces for three adjacent triangles, and in one case for four adjacent triangles, in order to get an intersection $\{0\}$. This is the subject of Section 4 (for $n>1$ ) and of Section 5 (for $n=1)$.

The equivalent formulation (c) of Theorem 1.1 raises the question of whether to estimate $\left(v, \Pi_{n}^{\mathcal{T}} v\right)_{\mathcal{T}}$ from below, and on the dependence of the triangle patch $\mathcal{T}$. Some generalities about this will be given in Section 6 .

In principle, all computations in this paper can be done by hand. Nevertheless, some of the more tedious computations we have done in Mathematica ${ }^{\circledR}$, while we have also checked many of the other computations using this program.

It is quite probable that the results and proofs in this paper can be carried over to the case that $\omega_{i}:=\left(a_{i} b_{i} c_{i}\right)^{\alpha}(\alpha>-1)$, i.e., that the weight function is some power of the product of the barycentric coordinates. We have refrained from doing the computations in this more general case because only the special case is needed in the application we have sketched. 


\section{Orthogonal Polynomials on the triangle}

Let $\alpha, \beta>-1$. The Jacobi polynomial $P_{n}^{(\alpha, \beta)}$ (see for instance [20]) is a polynomial of degree $n$ such that

$$
\int_{-1}^{1} P_{n}^{(\alpha, \beta)}(x) q(x)(1-x)^{\alpha}(1+x)^{\beta} d x=0
$$

for all polynomials $q$ of degree less than $n$, and

$$
P_{n}^{(\alpha, \beta)}(1)=\frac{(\alpha+1)_{n}}{n !} .
$$

Here the shifted factorial is defined by $(a)_{n}:=a(a+1) \ldots(a+n-1)$ for $n>0$ and $(a)_{0}:=1$. All zeros of $P_{n}^{(\alpha, \beta)}$ lie in $(-1,1)$, so it has definite sign on $[1, \infty)$ and on $(-\infty,-1]$.

The Jacobi polynomial has an explicit expression in terms of a terminating Gauss hypergeometric series

$$
{ }_{2} F_{1}\left(\begin{array}{c}
-n, b \\
c
\end{array} ; z\right):=\sum_{k=0}^{n} \frac{(-n)_{k}(b)_{k}}{(c)_{k} k !} z^{k}
$$

as follows:

$$
P_{n}^{(\alpha, \beta)}(x)=\frac{(\alpha+1)_{n}}{n !}{ }_{2} F_{1}\left(\begin{array}{c}
-n, n+\alpha+\beta+1 \\
\alpha+1
\end{array} ; \frac{1-x}{2}\right) .
$$

There is the symmetry relation

$$
P_{n}^{(\alpha, \beta)}(-x)=(-1)^{n} P_{n}^{(\beta, \alpha)}(x) .
$$

Let $T_{1}$ be the open (unit) triangle

$$
T_{1}:=\{(x, y) \mid x, y, 1-x-y>0\} .
$$

Let $\alpha, \beta, \gamma>-1$. Define in terms of Jacobi polynomials the bivariate polynomial

$$
P_{n, k}^{(\alpha, \beta, \gamma)}(x, y):=(1-x)^{k} P_{n-k}^{(\alpha, \beta+\gamma+2 k+1)}(1-2 x) P_{k}^{(\beta, \gamma)}\left(1-\frac{2 y}{1-x}\right) .
$$

This is a polynomial of degree $n$ in $x$ and $y$. For $(n, k) \neq(m, j)$ we have the orthogonality relation

$$
\int_{T_{1}} P_{n, k}^{(\alpha, \beta, \gamma)}(x, y) P_{m, j}^{(\alpha, \beta, \gamma)}(x, y) w_{\alpha, \beta, \gamma}(x, y) d x d y=0,
$$

where $w_{\alpha, \beta, \gamma}(x, y):=x^{\alpha} y^{\beta}(1-x-y)^{\gamma}$. This follows immediately from the orthogonality relations for Jacobi polynomials if we write

$$
\begin{aligned}
\int_{T_{1}} f(x, y) d x d y=\int_{0}^{1}\left(\int_{0}^{1-x} f(x, y) d y\right) d x & \\
& =\int_{0}^{1}(1-x)\left(\int_{0}^{1} f(x,(1-x) t) d t\right) d x .
\end{aligned}
$$

Thus with respect to the inner product for $L^{2}\left(T_{1}, x^{\alpha} y^{\beta}(1-x-y)^{\gamma} d x d y\right)$ the system $\left\{P_{m, j}^{(\alpha, \beta, \gamma)}\right\}_{0 \leq j \leq m \leq n}$ is an orthogonal basis of $\mathbb{P}_{n}\left(T_{1}\right)$.

These bivariate orthogonal polynomials on the triangle were introduced by Proriol [17]; see also the survey [15] and the monograph [12]. In the context of numerical 
analysis they were rediscovered in special cases in [11 and they got ample coverage in the monograph [14.

Denote by $\mathbb{P}_{n-1}^{\perp}\left(T_{1}\right)$ the orthoplement of $\mathbb{P}_{n-1}\left(T_{1}\right)$ in $\mathbb{P}_{n}\left(T_{1}\right)$ with respect to the inner product just mentioned. (So $\mathbb{P}_{-1}^{\perp}\left(T_{1}\right)=\mathbb{P}_{0}\left(T_{1}\right)$ consists of the constant functions.) Then the system $\left\{P_{n, j}^{(\alpha, \beta, \gamma)}\right\}_{0 \leq j \leq n}$ is a basis of $\mathbb{P}_{n-1}^{\perp}\left(T_{1}\right)$. In particular, the polynomial $(x, y) \mapsto P_{n}^{(\alpha, \beta+\gamma+1)}(1-2 x)$ is in $\mathbb{P}_{n-1}^{\perp}\left(T_{1}\right)$.

The symmetric group $S_{3}$ naturally acts on $T_{1}$. By considering the action of $S_{3}$ on (2.3) we obtain five further orthogonal bases for $\mathbb{P}_{n}$ with respect to the inner product for $L^{2}\left(T_{1}, x^{\alpha} y^{\beta}(1-x-y)^{\gamma} d x d y\right)$. The six bases are as follows (considered as functions of $(x, y))$ :

$$
\begin{array}{ll}
\left\{P_{m, j}^{(\alpha, \beta, \gamma)}(x, y)\right\}_{0 \leq j \leq m \leq n}, & \left\{P_{m, j}^{(\beta, \alpha, \gamma)}(y, x)\right\}_{0 \leq j \leq m \leq n}, \\
\left\{P_{m, j}^{(\beta, \gamma, \alpha)}(y, 1-x-y)\right\}_{0 \leq j \leq m \leq n}, & \left\{P_{m, j}^{(\alpha, \gamma, \beta)}(x, 1-x-y)\right\}_{0 \leq j \leq m \leq n}, \\
\left\{P_{m, j}^{(\gamma, \alpha, \beta)}(1-x-y, x)\right\}_{0 \leq j \leq m \leq n}, & \left\{P_{m, j}^{(\gamma, \beta, \alpha)}(1-x-y, y)\right\}_{0 \leq j \leq m \leq n} .
\end{array}
$$

In particular, each of these systems, when only taken for $m=n, 0 \leq j \leq n$, is an orthogonal basis for $\mathbb{P}_{n-1}^{\perp}\left(T_{1}\right)$. In combination with (2.2) this shows that the following polynomials in $(x, y)$ are elements of $\mathbb{P}_{n-1}^{\perp}\left(T_{1}\right)$ :

$$
P_{n}^{(\alpha, \beta+\gamma+1)}(1-2 x), \quad P_{n}^{(\beta, \alpha+\gamma+1)}(1-2 y), \quad P_{n}^{(\gamma, \alpha+\beta+1)}(2(x+y)-1) .
$$

If $T$ is another open triangle in $\mathbb{R}^{2}$ and if $\Lambda$ is an affine transformation of $\mathbb{R}^{2}$ which maps $T$ onto $T_{1}$ then the polynomials $P_{n, k}^{(\alpha, \beta, \gamma)} \circ \Lambda$ are orthogonal on $T$ with respect to the weight function $w_{\alpha, \beta, \gamma} \circ \Lambda$. If $\alpha=\beta=\gamma$ then the inner product on $T$ is independent, up to constant factor, of the choice of $\Lambda$. In the sequel we will have $\alpha=\beta=\gamma=1$. Similarly, as for $T_{1}$, we denote by $\mathbb{P}_{n-1}^{\perp}(T)$ the orthoplement of $\mathbb{P}_{n-1}(T)$ in $\mathbb{P}_{n}(T)$ with respect to this inner product.

\section{The intersection of $n$-TH DEGREe orthogonal POLYNOMIAL SPACES FOR TWO ADJACENT TRIANGLES}

In this section we keep using the conventions and definitions of Section 2 for $\alpha=\beta=\gamma=1$, and we will compare the orthogonal polynomials on the triangle $T_{1}$ for the weight function $w_{1,1,1}$ with the orthogonal polynomials on the adjacent triangle

$$
K_{c, d}:=\operatorname{conv}^{\mathrm{o}}\left((1,0),(0,0),\left(\frac{-c}{d-c}, \frac{1}{d-c}\right)\right) \quad(c \neq d)
$$

for the weight function $w_{1,1,1} \circ \Lambda$, where $\Lambda$ is the affine map sending $K_{c, d}$ to $T_{1}$, which is given by

$$
\Lambda(x, y)=(x+c y,(d-c) y) .
$$

We will prove:

Theorem 3.1. For $n>2$ the intersection of the spaces of orthogonal polynomials of degree $n$ on $T_{1}$ and $K_{c, d}$, i.e., the space $\mathbb{P}_{n-1}^{\perp}\left(T_{1}\right) \cap \mathbb{P}_{n-1}^{\perp}\left(K_{c, d}\right)$, has dimension zero unless $c=0$ or $d=1$ or $d-c=1$ or $c=1, d=0$. If $c=0, d=1$ then the intersection trivially has dimension $n+1$. In all other exceptional cases the intersection has dimension 1.

For $n=2$ the intersection has dimension zero unless $c=0$ or $d=1$ or $d-c=$ \pm 1 . If $c=0, d=1$ then the intersection trivially has dimension 3. In all other exceptional cases the intersection has dimension 1. 
For $n=1$ the intersection has dimension 1 except in the trivial case $c=0$, $d=1$, when it has dimension 2 .

In the cases that the intersection has dimension 1, it is spanned by a polynomial $q_{n}^{(c, d)}(x, y)$ as follows:

$$
\begin{aligned}
& q_{n}^{(0, d)}(x, y)= P_{n}^{(1,3)}(1-2 x), \\
& q_{n}^{(c, 1)}(x, y)= P_{n}^{(1,3)}(2(x+y)-1), \\
& q_{n}^{(c, c+1)}(x, y)= P_{n}^{(1,3)}(1-2 y), \\
& q_{n}^{(1,0)}(x, y)=y^{-1}\left(P_{n+1}^{(1,1)}(1-2(x+y))-P_{n+1}^{(1,1)}(1-2 x)\right), \\
& q_{2}^{(c, c-1)}(x, y)=28\left(6 x^{2}+6 c x y+c(c+1) y^{2}\right) \\
& \quad-21(c+3)(2 x+c y)+3 c^{2}+15 c+18, \\
& q_{1}^{(c, d)}(x, y)=3(c-d+1) x+3 c y-2 c+d-1 .
\end{aligned}
$$

In the case $d-c=1$ the triangles $T_{1}$ and $K_{c, d}$ have nonempty open intersection, so for the application we have in mind the result for this case is not needed.

Observe that, for $n=2$, (3.6) agrees up to a constant factor with (3.2), (3.3), (3.5) for $c=0,2,1$, respectively.

For usage in the proof we pick from the orthogonal systems in (2.4) one particular orthogonal basis for $\mathbb{P}_{n-1}^{\perp}\left(T_{1}\right)$, and we also renormalize it. The resulting basis consists of the following polynomials $p_{n, k}(k=0, \ldots, n)$.

$$
\begin{aligned}
p_{n, k}(x, y) & :=\frac{P_{n, k}^{(1,1,1)}(y, x)}{P_{n, k}^{(1,1,1)}(0,0)} \\
& ={ }_{2} F_{1}\left(\begin{array}{c}
-n+k, n+k+5 \\
2
\end{array} y\right)(1-y)^{k}{ }_{2} F_{1}\left(\begin{array}{c}
-k, k+3 \\
2
\end{array} \frac{x}{1-y}\right) \\
& ={ }_{2} F_{1}\left(\begin{array}{c}
-n+k, n+k+5 \\
2
\end{array} y\right) \sum_{j=0}^{k} \frac{(-k)_{j}(k+3)_{j}}{(2)_{j} j !} x^{j}(1-y)^{k-j}
\end{aligned}
$$

Similarly, for an orthogonal basis of $\mathbb{P}_{n-1}^{\perp}\left(K_{c, d}\right)$ we will take the polynomials $(x, y) \mapsto p_{n, k}(x+c y,(d-c) y)(k=0, \ldots, n)$.

Proof of Theorem 3.1. By (2.5) the polynomials

$$
P_{n}^{(1,3)}(1-2 x), \quad P_{n}^{(1,3)}(1-2 y), \quad P_{n}^{(1,3)}(2(x+y)-1)
$$

in $(x, y)$ are elements of $\mathbb{P}_{n-1}^{\perp}\left(T_{1}\right)$, and the polynomials

$$
P_{n}^{(1,3)}(1-2(x+c y)), \quad P_{n}^{(1,3)}(1-2(d-c) y), \quad P_{n}^{(1,3)}(2(x+d y)-1)
$$

are elements of $\mathbb{P}_{n-1}^{\perp}\left(K_{c, d}\right)$. Hence for $c=0$ or $d=1$ or $d-c=1$ the intersection considered in the theorem has dimension at least 1 , and the polynomial given by (3.2), (3.3), (3.4), respectively, is in this intersection.

A general element in $\mathbb{P}_{n-1}^{\perp}\left(T_{1}\right)$ has the form $\sum_{k=0}^{n} \alpha_{k} p_{n, k}(x, y)$, and a general element in $\mathbb{P}_{n-1}^{\perp}\left(K_{c, d}\right)$ has the form $\sum_{k=0}^{n} \beta_{k} p_{n, k}(x+c y,(d-c) y)$. Hence each nonzero element in $\mathbb{P}_{n-1}^{\perp}\left(T_{1}\right) \cap \mathbb{P}_{n-1}^{\perp}\left(K_{c, d}\right)$ corresponds to a nontrivial solution of 
the homogeneous linear system of equations

$$
\begin{array}{r}
\operatorname{coeff}\left(\sum_{k=0}^{n}\left(\alpha_{k} p_{n, k}(x, y)-\beta_{k} p_{n, k}(x+c y,(d-c) y)\right), x^{r} y^{m}\right)=0 \\
(r, m=0,1, \ldots, n, r+m \leq n)
\end{array}
$$

of $\frac{1}{2}(n+1)(n+2)$ equations in the $2(n+1)$ unknowns $\alpha_{0}, \ldots, \alpha_{n}, \beta_{0}, \ldots, \beta_{n}$.

By (3.8) and (2.2) the $n+1$ equations in (3.12) involving the coefficient of $x^{r}$ $(r=0, \ldots, n)$ amount to

$$
\sum_{k=0}^{n}\left(\alpha_{k}-\beta_{k}\right) \frac{P_{k}^{(1,1)}(1-2 x)}{P_{k}^{(1,1)}(1)}=0
$$

which implies $\alpha_{k}=\beta_{k}(k=0, \ldots, n)$. So the system of equations (3.12) reduces to

$$
\begin{gathered}
\sum_{k=0}^{n} \alpha_{k} \operatorname{coeff}\left(p_{n, k}(x, y)-p_{n, k}(x+c y,(d-c) y), x^{r} y^{m}\right)=0 \\
(r=0, \ldots, n-1, m=1, \ldots, n, r+m \leq n),
\end{gathered}
$$

which are $\frac{1}{2} n(n+1)$ homogeneous linear equations in the $n+1$ unknowns $\alpha_{0}, \ldots, \alpha_{n}$.

First we consider the case $n=1$. From (3.9) we get that

$$
p_{1,0}(x, y)=1-3 y \quad p_{1,1}(x, y)=1-2 x-y .
$$

Then we have to solve $\alpha_{0}, \alpha_{1}$ from the equation

$$
\alpha_{0}(1-3 y)+\alpha_{1}(1-2 x-y)=\alpha_{0}(1-3(d-c) y)+\alpha_{1}(1-2 x-(d+c) y) .
$$

This yields

$$
3(d-c-1) \alpha_{0}+(d+c-1) \alpha_{1}=0,
$$

which has (if not $c=0, d=1$ ) a one-dimensional solution space spanned by $\left(\alpha_{0}, \alpha_{1}\right):=\left(\frac{1}{2}(1-d-c), \frac{3}{2}(d-c-1)\right)$. Then $q_{1}^{(c, d)}(x, y)$ given by (3.7) equals $\alpha_{0} p_{1,0}(x, y)+\alpha_{1} p_{1,1}(x, y)$.

Now let $n \geq 2$. The power series coefficients in the left-hand sides of the equations (3.13) can be computed by using (3.9). We can rewrite the system (3.13) as

$$
\frac{1}{r !} \sum_{k=r}^{n} \alpha_{k} f_{k, r, m}(c, d)=0 \quad(r=0, \ldots, n-1, m=1, \ldots, n, r+m \leq n),
$$

where

$$
\begin{aligned}
f_{k, r, m}(c, d)= & \sum_{i=\max (0, m-k+r)}^{\min (m, n-k)} \frac{(-n+k)_{i}(n+k+5)_{i}}{(2)_{i} i !} \\
& \times \frac{(-k)_{r}(k+3)_{r}(r-k)_{m-i}}{(2)_{r}(m-i) !}\left(1-(d-c)^{m}\right) \\
- & \sum_{i=}^{\min (m-1, n-k)} \frac{(-n+k)_{i}(n+k+5)_{i}}{(2)_{i} i !} \\
& \quad \sum_{j=r+1}^{\min (k, m+r-i)} \frac{(-k)_{j}(k+3)_{j}(j-k)_{m+r-i-j}}{(2)_{j}(j-r) !(m+r-i-j) !} c^{j-r}(d-c)^{m+r-j} .
\end{aligned}
$$


In particular,

$$
f_{r, r, m}(c, d)=\frac{(-1)^{r}(2 r+2) !}{(r+1)(r+2) !} \frac{(-n+r)_{m}(n+r+5)_{m}}{(2)_{m} m !}\left(1-(d-c)^{m}\right),
$$

which is nonzero (note that $0 \leq m \leq n-r$, hence $(-n+r)_{m} \neq 0$ ) except if $d=c+1$ or $d=c-1$ and $m$ even. Thus, for $d \neq c+1$ the system (3.14) has a subsystem

$$
\sum_{k=r}^{n} \alpha_{k} f_{k, r, 1}(c, d)=0 \quad(r=0, \ldots, n-1)
$$

with $f_{r, r, 1}(c, d) \neq 0$. Thus $\alpha_{n}$ successively determines $\alpha_{n-1}, \alpha_{n-2}, \ldots, \alpha_{0}$, by which the system (3.14) has a solution space of dimension at most 1 if $d \neq c+1$.

For $d=c+1$ we have $f_{r, r, m}(c, c+1)=0$, while

$$
f_{r+1, r, m}(c, c+1)=(-1)^{m+r+1} \frac{(n-r-1) !(n+r+6)_{m-1}}{(n-r-m) !(m-1) ! m !} \frac{(r+4)_{r+1}}{r+2} c .
$$

This is nonzero unless $c=0$, but if $c=0$ then $d=1$ and we are in the trivial case. Thus, for $d=c+1, c \neq 0$ we successively get from (3.15) together with $f_{r, r, m}(c, c+1)=0, f_{r+1, r, m}(c, c+1) \neq 0$ that $\alpha_{n}=0, \alpha_{n-1}=0, \ldots, \alpha_{1}=0$. So only $\alpha_{0}$ may be nonzero by which the system (3.14) has a solution space of dimension at most 1 . In the beginning of the proof we already saw that this dimension is at least 1 . This settles the case $d=c+1$ in the theorem.

In the next step we consider the cases $(r, m)=(n-1,1),(n-2,1),(n-2,2)$ of (3.14) (for $n=2$ these are all possible cases). This gives the following system of three homogeneous linear equations in $\alpha_{n}, \alpha_{n-1}, \alpha_{n-2}$ :

$$
\begin{aligned}
& -\frac{(-1)^{n}(c+d-1)(2 n+1) !}{(n-1) !(n+2) !} \alpha_{n}+\frac{2(-1)^{n}(c-d+1)(n+2)(2 n-1) !}{(n-1) !(n+1) !} \alpha_{n-1}=0, \\
& \frac{(-1)^{n}(c+(c+d-1) n)(2 n) !}{(n-2) !(n+2) !} \alpha_{n} \\
& \quad-\frac{(-1)^{n} 2^{2 n-1}(c(n+1)-(d-1)(n+3))\left(\frac{1}{2}\right)_{n}}{n(n+1)(n-2) !} \alpha_{n-1} \\
& \quad-\frac{2(-1)^{n}(c-d+1)(2 n+3)(2 n-3) !}{(n-2) ! n !} \alpha_{n-2}=0, \\
& -\frac{(-1)^{n}\left(2 c d+\left((c+d)^{2}-1\right) n\right)(2 n) !}{2(n-2) !(n+2) !} \alpha_{n} \\
& \quad+\frac{(-1)^{n}\left(c^{2}-d^{2}+1\right)(n+2)(2 n-1) !}{(n-2) !(n+1) !} \alpha_{n-1} \\
& -\frac{2(-1)^{n}\left((c-d)^{2}-1\right)(n+2)(2 n+3)(2 n-3) !}{3(n-2) ! n !} \alpha_{n-2}=0 .
\end{aligned}
$$

The $3 \times 3$ determinant of the coefficients of the system (3.16) can be computed to be equal to

$$
\frac{(-1)^{n+1} 2^{4 n}(2 n) !\left(\frac{1}{2}\right)_{n-1}\left(\frac{1}{2}\right)_{n+2}}{3(n-2) ! n !((n+1) !)^{2}} c(d-1)(c-d+1)(c-d-1) .
$$

Thus $\alpha_{n}=\alpha_{n-1}=\alpha_{n-2}=0$ if not $c=0$ or $d=1$ or $c-d= \pm 1$. Together with (3.15) and $f_{r, r, 1}(c, d) \neq 0$ this implies that all $\alpha_{k}$ are zero if not $c=0$ or $d=1$ or 
$c-d= \pm 1$. This settles the theorem in the nonexceptional case except if $c-d=1$. For $c=0$ or $d=1$ the theorem is also settled now because we already observed in the beginning of the proof that the solution space has dimension at least 1 in these cases.

Now consider the case $d=c-1$. Then the third equation in (3.16) is a multiple of the first equation, so we can solve from the first and second equation of (3.16) that

$$
\begin{aligned}
\alpha_{n-1} & =\frac{(c-1) n(2 n+1)}{(n+2)^{2}} \alpha_{n}, \\
\alpha_{n-2} & =\frac{(n-1) n(2 n-1)(3(n+1)+c(c-2)(2 n+1))}{(n+1)(n+2)^{2}(2 n+3)} \alpha_{n} .
\end{aligned}
$$

For $n=2$ we conclude that the intersection has dimension 1 and that it contains the polynomial $p_{2,2}(x, y)+\alpha_{1} p_{2,1}(x, y)+\alpha_{0} p_{2,0}(x, y)$ with $\alpha_{1}$ and $\alpha_{0}$ given by (3.17) for $n=2$ and $\alpha_{2}=1$. Together with (3.9) this yields (3.6).

For $n>2$ we plug the above two equations into the cases $(r, m)=(n-3,1),(n-$ $3,2),(n-3,3)$ of (3.14) with $d=c-1$. The result is three homogeneous linear equations in $\alpha_{n}, \alpha_{n-3}$ of which the one for $m=2$ is trivial and of which the other two yield $\alpha_{n}=\alpha_{n-3}=0$ unless $c=0,1,2$. Again, together with (3.15) and $f_{r, r, 1}(c, d) \neq 0$ this implies that all $\alpha_{k}$ are zero if not $c=0,1,2$. For $c=0$ and for $c=2$ implying $d=1$ we already saw that the solution space has dimension 1 .

So the only remaining case to be considered is $(c, d)=(1,0)$. We will show that $q_{n}(x, y):=q_{n}^{(1,0)}(x, y)$, given by (3.5), clearly a polynomial of degree $n$, yields a (nonzero) element $q_{n}$ of $\mathbb{P}_{n-1}^{\perp}\left(T_{1}\right) \cap \mathbb{P}_{n-1}^{\perp}\left(K_{c, d}\right)$. By (2.5) and (2.2) we see that $(x, y) \mapsto P_{n+1}^{(1,1)}(1-2 x)$ is an orthogonal polynomial of degree $n+1$ on $T_{1}$ with respect to the weight function $x$ and that $(x, y) \mapsto P_{n+1}^{(1,1)}(1-2(x+y))=(-1)^{n} P_{n+1}^{(1,1)}(2(x+$ $y)-1$ ) is an orthogonal polynomial of degree $n+1$ on $T_{1}$ with respect to the weight function $1-x-y$. Then it holds for any polynomial $r(x, y)$ of degree $<n$ that

$$
\begin{aligned}
& \iint_{T_{1}} q_{n}(x, y) r(x, y) x y(1-x-y) d x d y \\
&=\iint_{T_{1}} P_{n+1}^{(1,1)}(1-2(x+y))(1-x-y) r(x, y) x d x d y \\
& \quad-\iint_{T_{1}} P_{n+1}^{(1,1)}(1-2 x) x r(x, y)(1-x-y) d x d y=0-0=0 .
\end{aligned}
$$

Hence $q_{n} \in \mathbb{P}_{n}^{\perp}\left(T_{1}\right)$. Since $q_{n}$ is invariant under the transformation $(x, y) \mapsto(x+$ $y,-y)$, we have $q_{n} \in \mathbb{P}_{n}^{\perp}\left(K_{1,0}\right) \cap \mathbb{P}_{n}^{\perp}\left(T_{1}\right)$. Since we already proved that in this case the intersection has dimension at most one, we are finished.

\section{The InTERSECTION OF $n$-TH DEGREE ORTHOGONAL POLYNOMIAL SPACES FOR A TRIANGLE PATCH (CASE $n>1)$}

Since polynomial spaces $\mathbb{P}_{n}(D)$ are invariant under affine coordinate transformations, it suffices to prove Theorem 1.1 for a reference configuration with center $z:=(0,0)$ and with one of the triangles, say $K_{i}$, equal to the unit triangle $T_{1}$ given by (2.1). Hence, the adjacent triangle $K_{i+1}$ to the left of $K_{i}$ lies in the left half-plane while the other one, i.e., $K_{i-1}$ lies in the lower half-plane. See Figure 2. 
In this Section we will prove the intersection property Theorem 1.1 (b) for polynomial degrees $n>1$. First we will describe the exceptional cases in Theorem 3.1 in terms of geometric quantities. For this we introduce the "critical sets" for a triangle; for an illustration see Figure 3 .

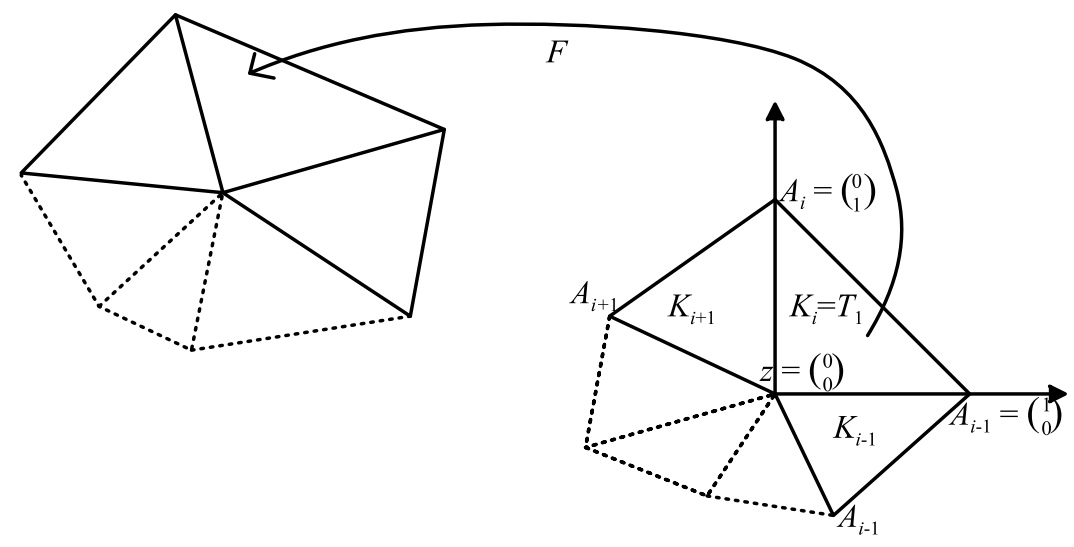

FiguRE 2. Reference configuration. $K_{i}=T_{1}$ is the unit triangle, $K_{i+1}$ is in the left half-plane and $K_{i-1}$ in the lower half-plane.

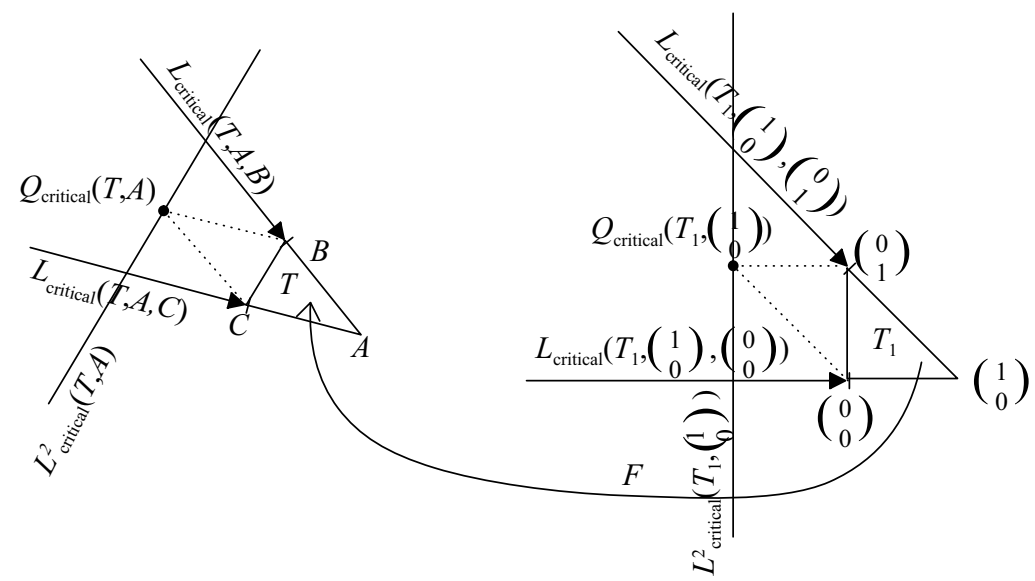

Figure 3. Critical sets for the triangle $T$ and critical sets for the unit triangle. 
Definition 4.1. For a triangle $T$ with vertices 2 $A, B, C$, the critical sets with respect to two vertices $A, B$ are

$$
\begin{aligned}
& \mathcal{L}_{\text {critical }}(T, A, B):=\{A+t(B-A): t \geq 1\}, \\
& Q_{\text {critical }}(T, A):=B+C-A, \\
& \mathcal{L}_{\text {critical }}^{2}(T, A):=\{2 B-A+t(C-B): t \in \mathbb{R}\}, \\
& \mathcal{L}_{\text {critical }}^{n, \text { tot }}(T, A):=\mathcal{L}_{\text {critical }}(T, A, B) \cup \mathcal{L}_{\text {critical }}(T, A, C) \cup\left\{Q_{\text {critical }}(T, A)\right\}(n>2), \\
& \mathcal{L}_{\text {critical }}^{2 \text { tot }}(T, A):=\mathcal{L}_{\text {critical }}(T, A, B) \cup \mathcal{L}_{\text {critical }}(T, A, C) \cup \mathcal{L}_{\text {critical }}^{2}(T, A)(n=2) .
\end{aligned}
$$

Note that an orientation preserving affine map sending a triangle $T$ to a triangle $\tilde{T}$ maps the critical sets of $T$ to the corresponding critical sets of $\tilde{T}$. Also note that, for $n=2, \mathcal{L}_{\text {critical }}^{2}(T, A)$ contains $Q_{\text {critical }}(T, A)$ and intersects with $\mathcal{L}_{\text {critical }}(T, A, B)$ and with $\mathcal{L}_{\text {critical }}(T, A, C)$.

Proposition 4.2. Let $K_{1}=\operatorname{conv}^{\mathrm{o}}(A, B, C)$ and $K_{2}=\operatorname{conv}^{\mathrm{o}}(B, A, D)$ be two disjoint triangles with common edge $A B$. Then for $n>1$ :

$$
\operatorname{dim}\left(\mathbb{P}_{n-1}^{\perp}\left(K_{1}\right) \cap \mathbb{P}_{n-1}^{\perp}\left(K_{2}\right)\right)= \begin{cases}0 & \text { if } D \notin \mathcal{L}_{\text {critical }}^{n, \text { tot }}\left(K_{1}, C\right), \\ 1 & \text { if } D \in \mathcal{L}_{\text {critical }}^{n, \text { tot }}\left(K_{1}, C\right) .\end{cases}
$$

Proof. We consider first the case that $K_{1}=T_{1}$, and apply Theorem 3.1. Let $D=\left(\frac{-c}{d-c}, \frac{1}{d-c}\right)$ (cf. (3.1) ). The exceptional cases are given by

(1) $c=0$. Since $K_{1}$ and $K_{2}$ have disjoint interior this is equivalent to

$$
D \in \mathcal{L}_{\text {critical }}\left(T_{1},(0,1),(0,0)\right) .
$$

(2) $d=1$. Again, taking into account that $K_{1}$ and $K_{2}$ have empty open intersection we get that this case is equivalent to

$$
D \in \mathcal{L}_{\text {critical }}\left(T_{1},(0,1),(1,0)\right) .
$$

(3) $d-c=1$. This case contradicts the condition $K_{1} \cap K_{2}=\emptyset$ and, hence, cannot arise.

(4) $c=1, d=0$. This case is equivalent to $D=Q_{\text {critical }}\left(T_{1},(0,1)\right)=(1,-1)$.

(5) $n=2$ and $d=c-1$. Then $D=(s,-1)(s \in \mathbb{R})$, so this case is equivalent to $D \in \mathcal{L}_{\text {critical }}^{2}\left(T_{1},(0,1)\right)$.

The general case follows by employing an affine pullback of a general triangle $K_{1}=\operatorname{conv}^{\mathrm{o}}(A, B, C)$ to $T_{1}$ such that $C$ is sent to $(0,1)$.

Proof of Theorem 1.1(b) for $n>1$. We use the numbering of triangles, edges, vertices in $\mathcal{T}_{z}$ as in Figure 1. If there exists an edge $E_{i}$ with adjacent triangles $K_{i}$, $K_{i+1}$ such that

$$
A_{i+1} \notin \mathcal{L}_{\text {critical }}^{n, \text { tot }}\left(K_{i}, A_{i-1}\right) \quad \vee \quad A_{i-1} \notin \mathcal{L}_{\text {critical }}^{n, \text { tot }}\left(K_{i+1}, A_{i+1}\right)
$$

we conclude from Proposition 4.2 that

$$
\mathbb{P}_{n-1}^{\perp}\left(K_{i}\right) \cap \mathbb{P}_{n-1}^{\perp}\left(K_{i+1}\right)=\{0\}
$$

and the statement follows.

Hence, for the rest of the proof we always assume that

$$
\forall i \in\{1, \ldots, q\} A_{i+1} \in \mathcal{L}_{\text {critical }}^{n, \text { tot }}\left(K_{i}, A_{i-1}\right) \wedge A_{i-1} \in \mathcal{L}_{\text {critical }}^{n, \text { tot }}\left(K_{i+1}, A_{i+1}\right) .
$$

\footnotetext{
${ }^{2}$ As a convention we list the vertices $A, B, C$ of a triangle $T=\operatorname{conv}^{\circ}(A, B, C)$ always in the counterclockwise ordering.
} 


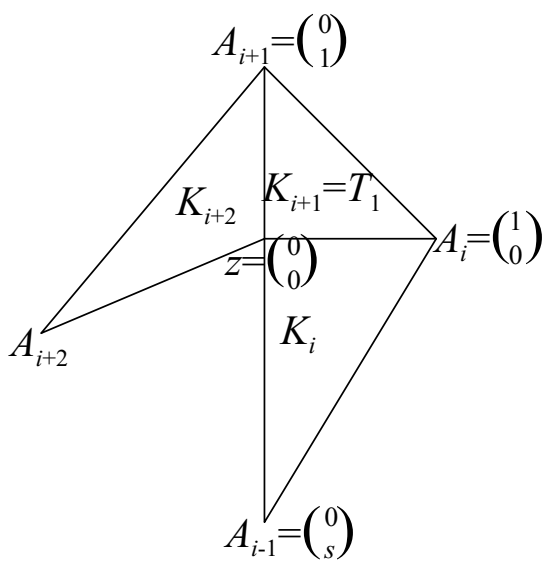

Figure 4. Illustration of Case a) in the proof of Theorem 1.1(b) for $n>1$.

Clearly we can pick a vertex $A_{i}$ such that the inner angle at $A_{i}$, (i.e., the angle $\angle A_{i-1} A_{i} A_{i+1}$, seen from $z$ ) is less than $\pi$. This property excludes that $A_{i+1} \in$ $\mathcal{L}_{\text {critical }}\left(K_{i}, A_{i-1}, A_{i}\right)$, or equivalently $A_{i-1} \in \mathcal{L}_{\text {critical }}\left(K_{i+1}, A_{i+1}, A_{i}\right)$. The property is also preserved under affine maps. We distinguish between the following cases.

Case a) $A_{i+1} \in \mathcal{L}_{\text {critical }}\left(K_{i}, A_{i-1}, z\right)$.

Without loss of generality we can work in the reference situation (cf. Figure 4) that $K_{i+1}=T_{1}$, i.e., $A_{i+1}=(0,1), z=(0,0)$ and $A_{i-1}=(0, s)$ for some $s<0$. From Theorem [3.1, in particular from (3.2), it follows that $\mathbb{P}_{n-1}^{\perp}\left(K_{i+1}\right) \cap \mathbb{P}_{n-1}^{\perp}\left(K_{i}\right)$ is spanned by the polynomial $q(x, y):=P_{n}^{(1,3)}(1-2 x)$. Note that the adjacent triangle $K_{i+2}$ left to $T_{1}$ lies in the left half-plane. Hence $q$ is either positive on $K_{i+2}$ or negative, by which it cannot be orthogonal to all constant functions on $K_{i+2}$. We conclude that

$$
\mathbb{P}_{n-1}^{\perp}\left(K_{i+2}\right) \cap \mathbb{P}_{n-1}^{\perp}\left(K_{i+1}\right) \cap \mathbb{P}_{n-1}^{\perp}\left(K_{i}\right)=\{0\} .
$$

Case b) $\quad A_{i+1}=Q_{\text {critical }}\left(K_{i}, A_{i-1}\right)$ and $A_{i-2} \in \mathcal{L}_{\text {critical }}\left(K_{i}, A_{i}, z\right)$.

Then $A_{i} \in \mathcal{L}_{\text {critical }}\left(K_{i-1}, A_{i-2}, z\right)$. So as in case a), but now with $K_{i}=T_{1}$, we conclude that

$$
\mathbb{P}_{n-1}^{\perp}\left(K_{i-1}\right) \cap \mathbb{P}_{n-1}^{\perp}\left(K_{i}\right) \cap \mathbb{P}_{n-1}^{\perp}\left(K_{i+1}\right)=\{0\} .
$$

Case c) $\quad A_{i+1}=Q_{\text {critical }}\left(K_{i}, A_{i-1}\right)$ and $A_{i-2} \in \mathcal{L}_{\text {critical }}\left(K_{i}, A_{i}, A_{i-1}\right)$.

Without loss of generality we can work in the reference configuration (see Figure 5) that $K_{i}=T_{1}$ with $A_{i-1}=(0,0), A_{i}=(1,0), z=(0,1)$. Then $K_{i+1}=$ $\operatorname{conv}^{\mathrm{o}}((1,0),(1,1),(0,1)), K_{i-1}=\operatorname{conv}^{\mathrm{o}}((0,0),(0,1),(s, 0))$ for some $s<0$, and $K_{i+2}=\operatorname{conv}^{\mathrm{o}}((0,1),(1,1),(t, u))$ with $u>1$. From Theorem [3.1 in particular from (3.4), we conclude that $\mathbb{P}_{n-1}^{\perp}\left(K_{i}\right) \cap \mathbb{P}_{n-1}^{\perp}\left(K_{i-1}\right)$ is spanned by the polynomial $r(x, y):=P_{n}^{(1,3)}(1-2 y)$. By arguing as in Case a) we conclude that $r$ is not changing sign in $K_{i+2}$ (since $\left.y \geq 1\right)$. Hence

$$
\mathbb{P}_{n-1}^{\perp}\left(K_{i-1}\right) \cap \mathbb{P}_{n-1}^{\perp}\left(K_{i}\right) \cap \mathbb{P}_{n-1}^{\perp}\left(K_{i+1}\right) \cap \mathbb{P}_{n-1}^{\perp}\left(K_{i+2}\right)=\{0\} .
$$




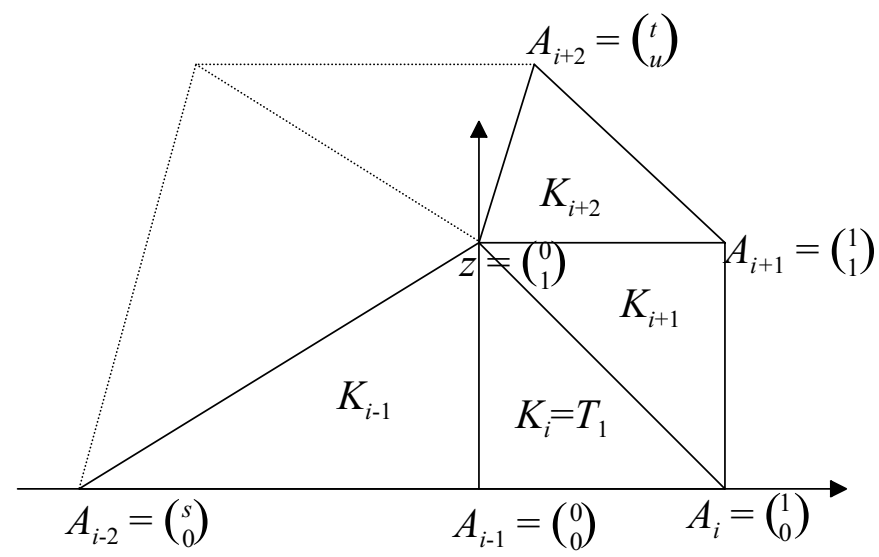

FiguRE 5. Illustration of the geometric argument for case c). The points $A_{i-2}, A_{i-1}, A_{i}$ are collinear and $\overline{K_{i} \cup K_{i+1}}$ form a parallelogram.

Case d) $\quad A_{i+1}=Q_{\text {critical }}\left(K_{i}, A_{i-1}\right)$ and $A_{i-2}=Q_{\text {critical }}\left(K_{i}, A_{i}\right)$.

Without loss of generality we can consider the reference situation that $K_{i}=T_{1}$ with $z=(0,0), A_{i-2}=(1,-1), A_{i-1}=(1,0), A_{i}=(0,1), A_{i+1}=(-1,1)$. From Theorem 3.1] we conclude that $\mathbb{P}_{n-1}^{\perp}\left(K_{i}\right) \cap \mathbb{P}_{n-1}^{\perp}\left(K_{i-1}\right)$ is spanned by the polynomial $q_{n}(x, y):=q_{n}^{(1,0)}(x, y)$ given by (3.5), and that $\mathbb{P}_{n-1}^{\perp}\left(K_{i+1}\right) \cap \mathbb{P}_{n-1}^{\perp}\left(K_{i}\right)$ is spanned by the polynomial $q_{n}(y, x)$. Since these two polynomials are linearly independent (compare the highest degree part of both polynomials), we have shown that

$$
\mathbb{P}_{n-1}^{\perp}\left(K_{i-1}\right) \cap \mathbb{P}_{n-1}^{\perp}\left(K_{i}\right) \cap \mathbb{P}_{n-1}^{\perp}\left(K_{i+1}\right)=\{0\} .
$$

Case e) $\quad A_{i+1} \in \mathcal{L}_{\text {critical }}^{2}\left(K_{i}, A_{i-1}\right)$ and $A_{i-2} \in \mathcal{L}_{\text {critical }}\left(K_{i}, A_{i}, A_{i-1}\right)$.

We can assume that $K_{i}=T_{1}$ with $z=(0,0)$. Then $A_{i+1}=(-1, c)$. From (3.6) we have that $\mathbb{P}_{n-1}^{\perp}\left(K_{i+1}\right) \cap \mathbb{P}_{n-1}^{\perp}\left(K_{i}\right)$ is spanned by the polynomial $q_{2}^{(c, c-1)}(y, x)$ and from (3.3) that $\mathbb{P}_{n-1}^{\perp}\left(K_{i}\right) \cap \mathbb{P}_{n-1}^{\perp}\left(K_{i-1}\right)$ is spanned by the polynomial $P_{n}^{(1,3)}(2(x+$ $y)-1)$. A computation shows that these two polynomials are linearly dependent iff $c=2$. But then the inner angle at $A_{i}$ equals $\pi$, which we excluded.

Case f) $\quad A_{i+1} \in \mathcal{L}_{\text {critical }}^{2}\left(K_{i}, A_{i-1}\right)$ and $A_{i-2} \in \mathcal{L}_{\text {critical }}\left(K_{i}, A_{i}, z\right)$.

Again assume that $K_{i}=T_{1}$ with $z=(0,0)$. Then $A_{i-2}=(0, s)$ for some $s<0$. We can argue as in case a), but now with $K_{i}=T_{1}$.

Case g) $\quad A_{i+1} \in \mathcal{L}_{\text {critical }}^{2}\left(K_{i}, A_{i-1}\right)$ and $A_{i-2} \in \mathcal{L}_{\text {critical }}^{2}\left(K_{i}, A_{i}\right)$.

Again assume that $K_{i}=T_{1}$ with $z=(0,0)$. Then $A_{i+1}=(-1, c)$ and $A_{i-2}=$ $(d,-1)$ for some $c, d \in \mathbb{R}$. From (3.6) we have that $\mathbb{P}_{n-1}^{\perp}\left(K_{i+1}\right) \cap \mathbb{P}_{n-1}^{\perp}\left(K_{i}\right)$ is spanned by the polynomial $q_{2}^{(c, c-1)}(y, x)$ and that $\mathbb{P}_{n-1}^{\perp}\left(K_{i}\right) \cap \mathbb{P}_{n-1}^{\perp}\left(K_{i-1}\right)$ is spanned by the polynomial $q_{2}^{(d, d-1)}(x, y)$. A computation shows that these two polynomials are linearly dependent iff $c=d=-3$ or $c=d=2$. But in the first case the triangles $K_{i-1}$ and $K_{i+1}$ intersect, and in the second case the inner angle at $A_{i}$ equals $\pi$. 

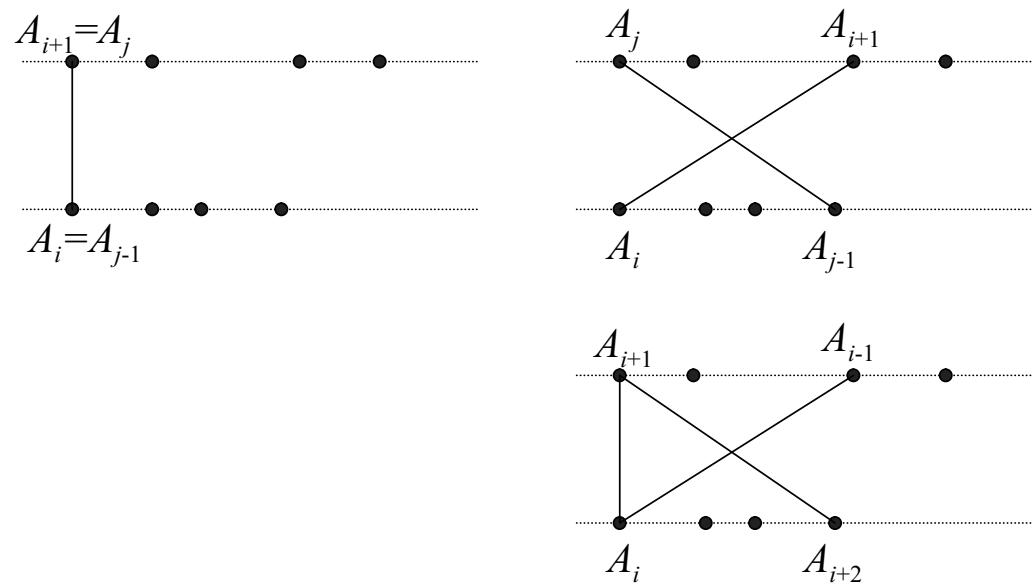

FiguRE 6. Illustration of the last part of the proof of Lemma 5.1 ( $q$ even). $A_{i+1}=A_{j}$ (left picture) because otherwise the top right picture gives a contradiction. But now the bottom picture gives a contradiction.

\section{The intersection of $n$-TH DEGREE ORTHOGONAL POLYNOMIAL SPACES FOR A TRIANGLE PATCH (CASE $n=1)$}

In this section we will prove the intersection property Theorem 1.1(b) polynomial degrees $n=1$. We need two simple lemmas.

Lemma 5.1. Let $\mathcal{T}:=\left\{K_{i}: 1 \leq i \leq q\right\}$ denote a triangle patch around $z \in \mathbb{R}^{2}$. Then there are $K_{i-1}, K_{i}, K_{i+1}$ of which the barycenters are not collinear.

Proof. Let $M_{i}$ be the barycenter of $K_{i}$. We will show that the points $M_{i}(i=$ $1, \ldots, q)$ cannot be collinear. This will imply the statement of the lemma.

We may choose $z=(0,0)$. Suppose that the points $M_{i}=\frac{1}{3}\left(A_{i-1}+A_{i}\right)$ $(i=1, \ldots, q)$ are collinear. Then all vectors $3\left(M_{i}-M_{i-1}\right)=A_{i}-A_{i-2}$ are proportional. If $q$ is odd then this implies that all vertices $A_{i}$ are collinear, which is impossible. If $q$ is even then the set of vertices $A_{1}, A_{3}, \ldots, A_{q-1}$ and the set of vertices $A_{2}, A_{4}, \ldots, A_{q}$ are both collinear and the two collinear sets lie on parallel lines. Since all vertices cannot be collinear, these two parallel lines have to be distinct. After applying an affine linear map we may assume that one of the lines is $y=0$ with $A_{i}=(0,0)$ for some $i$ and with all other vertices on this line having coordinates $(x, 0)$ with $x>0$, and that the other line is $y=1$ with $A_{j}=(0,1)$ for some $j \neq i$ and with all other vertices on this line having coordinates $(x, 1)$ with $x>0$. First we show that $j=i+1$. Indeed, if $j \neq i+1$ then the edge connecting $A_{j}$ and $A_{j-1}$ will cross the edge connecting $A_{i}$ and $A_{i+1}$, which is not allowed. Thus $A_{i+1}=(0,1)$. But now the edge connecting $A_{i+1}$ and $A_{i+2}$ will cross the edge connecting $A_{i}$ and $A_{i-1}$, which is not allowed (see Figure 6 for this last part of the proof, where we successively arrive twice at a contradiction). Thus we cannot have two collinear sets of vertices if $q$ is even.

Lemma 5.2. Let $K$ be a triangle with barycenter $M$. Let $\mu$ be a finite measure on $K$ which is invariant under all affine transformations mapping $K$ onto itself (these 
form a group ismorphic to $\left.S_{3}\right)$. Then

$$
\int_{K} p d \mu=p(M) \mu(K) \quad \text { for all } p \in \mathbb{P}_{1}(K)
$$

This holds, in particular, if $d \mu(x, y)=\omega(x, y) d x d y$ with $\omega$ the product of the barycentric coordinates for $K$.

Proof. Let $A, B, C$ be the vertices of $K$. Since the assertion is trivial for constant functions, it is sufficient to prove the property for affine linear functions $p$ vanishing on one of the medians $A M, B M, C M$. Suppose $p$ vanishes on $A M$. Then the function $p-p(M)$ is sent to its opposite under the affine map fixing $A$ and interchanging $B$ and $C$ (check this for the reference triangle $T$ with $A=(0,0)$, $B=(1,0), C=(0,1))$. Hence $\int_{K}(p-p(M)) d \mu=0$.

Proof of Theorem 1.1(b) for $n=1$. Let $\mathcal{T}:=\left\{K_{i}: 1 \leq i \leq q\right\}$ denote a triangle patch around a point $z \in \mathbb{R}^{2}$ and let $\Omega:=\cup_{i=1}^{q} \overline{K_{i}}$. By Lemma 5.1 there are $K_{i-1}, K_{i}, K_{i+1}$ such that their barycenters $M_{i-1}, M_{i}, M_{i+1}$ are not collinear. Now suppose that $u \in \mathbb{P}_{1}(\Omega)$ and $u \in \mathbb{P}_{0}^{\perp}\left(K_{j}\right)$ for $j=i-1, i, i+1$. By Lemma 5.2 we have for $j \in\{i-1, i, i+1\}$ that

$$
0=\frac{\int_{K_{j}} u(x, y) \omega_{j}(x, y) d x d y}{\int_{K_{j}} \omega_{j}(x, y) d x d y}=u\left(M_{j}\right)
$$

Since the affine linear function $u$ vanishes on three points which are not collinear, $u$ is identically zero.

Remark 5.3. If we take the triangle patch such that $K_{i}=T_{1}$ with $z=(0,0)$, $A_{i-1}=(1,0), A_{i}=(0,1), A_{i-2}=\left(\frac{-c}{d-c}, \frac{1}{d-c}\right)$ and $A_{i+1}=\left(\frac{1}{d^{\prime}-c^{\prime}}, \frac{-c^{\prime}}{d^{\prime}-c^{\prime}}\right)$, then we see from (3.7) that

$$
\mathbb{P}_{0}^{\perp}\left(K_{i-1}\right) \cap \mathbb{P}_{0}^{\perp}\left(K_{i}\right) \cap \mathbb{P}_{0}^{\perp}\left(K_{i+1}\right) \neq\{0\}
$$

implies that the polynomials $q_{1}^{(c, d)}(x, y)$ and $q_{1}^{\left(c^{\prime}, d^{\prime}\right)}(y, x)$ are multiples of each other. A computation shows that then

$$
c^{\prime}=k(c-d+1), \quad d^{\prime}=k(1-d)+1 \quad(0 \neq k \in \mathbb{R}) .
$$

Hence $A_{i+1}=\left(\frac{1}{1-k c}, \frac{k(d-c-1)}{1-k c}\right)$. Then $A_{i+1}-A_{i-1}$ and $A_{i}-A_{i-2}$ are proportional. By the Proof of Lemma 5.1 this gives the collinearity of the barycenters of $K_{i-1}$, $K_{i}$ and $K_{i+1}$. Thus we have shown once more that, if the barycenters of these three triangles are not collinear, then $\mathbb{P}_{0}^{\perp}\left(K_{i-1}\right) \cap \mathbb{P}_{0}^{\perp}\left(K_{i}\right) \cap \mathbb{P}_{0}^{\perp}\left(K_{i+1}\right)=\{0\}$. 


\section{INJECTIVITY FOR THE POLYNOMIAL PROJECTION OPERATOR: SOME FOLLOW-UP}

Recall Theorem 1.1(c) about the injectivity of the polynomial projection operator $\Pi_{n}^{\mathcal{T}}: \mathbb{P}_{n}(\Omega) \rightarrow \mathbb{P}_{n-1}(\mathcal{T})$. Let $\|\cdot\|_{\mathcal{T}}:=(\cdot, \cdot)_{\mathcal{T}}^{1 / 2}$ and define

$$
\begin{aligned}
& c_{n}^{\prime}(\mathcal{T}):=\inf _{v \in \mathbb{P}_{n}(\Omega) \backslash\{0\}} \frac{\left(v, \Pi_{n}^{\mathcal{T}} v\right)_{\mathcal{T}}}{(v, v)_{\mathcal{T}}}=\inf _{v \in \mathbb{P}_{n}(\Omega) \backslash\{0\}} \frac{\left\|\Pi_{n}^{\mathcal{T}} v\right\|_{\mathcal{T}}^{2}}{\|v\|_{\mathcal{T}}^{2}}>0, \\
& c_{n}^{\prime \prime}(\mathcal{T}):=\inf _{v \in \mathbb{P}_{n}(\Omega) \backslash\{0\}} \frac{\|v\|_{\mathcal{T}}^{2}}{\|v\|_{L^{2}(\Omega)}^{2}}>0, \\
& \check{c}_{n}(\mathcal{T}):=\inf _{v \in \mathbb{P}_{n}(\Omega) \backslash\{0\}} \frac{\left(v, \Pi_{n}^{\mathcal{T}} v\right)_{\mathcal{T}}}{(v, v)_{L^{2}(\Omega)}} \geq c_{n}^{\prime \prime}(\mathcal{T}) c_{n}^{\prime}(\mathcal{T})>0 .
\end{aligned}
$$

The inequality in (6.1) follows from the injectivity of $\Pi_{n}^{\mathcal{T}}$, while the inequality in (6.2) is a consequence of the equivalence of all norms on a finite dimensional space.

Remark 6.1. We can expand the squared norms in (6.1) and (6.2). Let $\Lambda_{i}$ be the orientation preserving affine linear map of $T_{1}$ onto $K_{i}$ which sends $(0,0)$ to $z$. Let $\left\{p_{m}\right\}_{m=1, \ldots, n(n+1) / 2}$ be an orthonormal basis for $\mathbb{P}_{n-1}\left(T_{1}\right)$ with respect to the weight function $\omega$ on $T_{1}$. Then

$$
\begin{gathered}
\left\|\Pi_{n}^{\mathcal{T}} v\right\|_{\mathcal{T}}^{2}=\sum_{i=1}^{q} \operatorname{det} \Lambda_{i} \sum_{m=1}^{\frac{1}{2} n(n+1)}\left(\int_{T_{1}} v \circ \Lambda_{i} p_{m} \omega\right)^{2}, \\
\|v\|_{\mathcal{T}}^{2}=\sum_{i=1}^{q} \operatorname{det} \Lambda_{i} \int_{T_{1}}\left(v \circ \Lambda_{i}\right)^{2} \omega, \quad\|v\|_{L^{2}(\Omega)}^{2}=\sum_{i=1}^{q} \operatorname{det} \Lambda_{i} \int_{T_{1}}\left(v \circ \Lambda_{i}\right)^{2} .
\end{gathered}
$$

Hence $c_{n}^{\prime \prime}=c_{n}^{\prime \prime}(\mathcal{T})$ is independent of the choice of the patch $\mathcal{T}$ and it equals

$$
c_{n}^{\prime \prime}=\inf _{v \in \mathbb{P}_{n}\left(T_{1}\right) \backslash\{0\}} \frac{\int_{T_{1}} v(x, y)^{2} x y(1-x-y) d x d y}{\int_{T_{1}} v(x, y)^{2} d x d y} .
$$

Remark 6.2. From [21, Prop. 3.46] there follows an $n$-explicit lower bound for $c_{n}^{\prime \prime}$ as given by (6.4): $c_{n}^{\prime \prime} \geq C /(n+1)^{4}$ with a fixed $n$-independent constant $C>0$.

Since the quotients of integrals in (6.1) -6.3 are invariant under affine linear maps, we might restrict to the case that $K_{1}$ is the reference triangle $T_{1}$. But in view of the numerical applications, we consider only translations and rotations of triangle patches. Thus we restrict in the following to the case that $z=0$ and $A_{q}=\left(r_{q}, 0\right)$ for some $r_{q}>0$. The further data determining $\mathcal{T}$ are, for $i=1, \ldots, q$, the angles $\alpha_{i}, \beta_{i}, \gamma_{i}$ of the triangle $K_{i}$ at $z, A_{i-1}, A_{i}$, respectively, together with the length $r_{i}$ of the edge connecting $z$ with $A_{i}$ (see Figure 7). Evident constraints on these numbers are that $\alpha_{i}+\beta_{i}+\gamma_{i}=\pi$ and $\alpha_{1}+\cdots+\alpha_{q}=2 \pi$. But $\mathcal{T}$ would already be completely determined by $\alpha_{2}, \ldots, \alpha_{q}, \beta_{2}, \ldots, \beta_{q}$ and $r_{q}$, or by $\alpha_{2}, \ldots, \alpha_{q}$ and $r_{1}, \ldots, r_{q}$. The map $\left(\alpha_{2}, \ldots, \alpha_{q}, \beta_{2}, \ldots, \beta_{q}, r_{q}\right) \leftrightarrow\left(\alpha_{2}, \ldots, \alpha_{q}, r_{1}, \ldots, r_{q}\right)$ is continuous in both directions, as can be seen from the following identities obtained by a combination of the sine rule and the cosine rule for the triangle $K_{i}$ :

$$
\frac{\sin \alpha_{i}}{\sqrt{r_{i-1}^{2}+r_{i}^{2}-2 r_{i-1} r_{i} \cos \alpha_{i}}}=\frac{\sin \beta_{i}}{r_{i}}=\frac{\sin \left(\pi-\alpha_{i}-\beta_{i}\right)}{r_{i-1}} .
$$




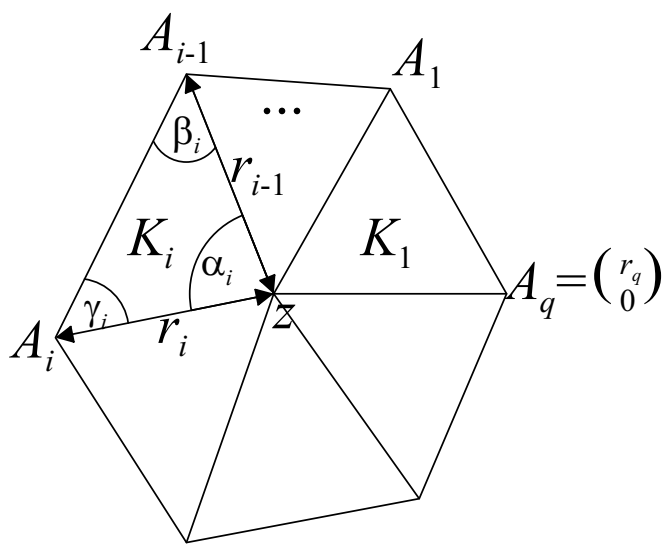

FIGURE 7. Illustration of angles $\alpha_{1}, \beta_{i}, \gamma_{i}$, and radii $r_{i}$ for a triangle patch.

The quotients of integrals in (6.1)-6.3 will depend continuously on $v$ and the data of $\mathcal{T}$. Therefore, the three constants in (6.1)-6.3) will depend continuously on the data of $\mathcal{T}$ and they will remain bounded away from zero if we let the data of $\mathcal{T}$ range over a compact set. To fix a compact set, choose $\delta \in(0, \pi / 3]$ and $\rho>0$.

Definition 6.3. The compact set of triangle patches $X_{q, \delta, \rho}$ consists of all $\mathcal{T}$ with $\alpha_{i}, \beta_{i}, \gamma_{i} \geq \delta$ and $r_{i} \geq \rho(i=1, \ldots, q)$. Furthermore, define

$$
\tilde{c}_{n}(q, \delta, \rho):=\inf _{\mathcal{T} \in X_{q, \delta, \rho}} \check{c}_{n}(\mathcal{T}) .
$$

By the second equality in (6.5) we see that, for given $\delta$ and $q$ there exists $C>0$ such that $\rho \leq r_{i} \leq C \rho(i=1, \ldots, q)$ if $\mathcal{T} \in X_{q, \delta, \rho}$. Since the quotients of integrals in (6.1) - (6.3) are invariant under dilations, $\tilde{c}_{n}(q, \delta, \rho)$ will be independent of $\rho$. Since necessarily $q \delta \leq 2 \pi, X_{q, \delta, \rho}$ is nonempty for only finitely many values of $q$. We conclude:

Theorem 6.4. $\inf _{\rho>0, q \geq 3} \tilde{c}_{n}(q, \delta, \rho)>0$.

\section{REFERENCES}

[1] Mark Ainsworth and J. Tinsley Oden, A Posteriori Error Estimation in Finite Element Analysis, Pure and Applied Mathematics (New York), Wiley-Interscience [John Wiley \& Sons], New York, 2000. MR.1885308 (2003b:65001)

[2] I. Babuška and W. C. Rheinboldt, A-posteriori error estimates for the finite element method, Internat. J. Numer. Meth. Engrg. 12 (1978), 1597-1615.

[3] I. Babuška and W. C. Rheinboldt, Error estimates for adaptive finite element computations, SIAM J. Numer. Anal. 15 (1978), no. 4, 736-754. MR0483395 (58 \#3400)

[4] Wolfgang Bangerth and Rolf Rannacher, Adaptive Finite Element Methods for Differential Equations, Lectures in Mathematics ETH Zürich, Birkhäuser Verlag, Basel, 2003. MR1960405 (2004b:65002)

[5] R. E. Bank and A. Weiser, Some a posteriori error estimators for elliptic partial differential equations, Math. Comp. 44 (1985), no. 170, 283-301, DOI 10.2307/2007953. MR777265 (86g:65207)

[6] Randolph E. Bank and Jinchao Xu, Asymptotically exact a posteriori error estimators. II. General unstructured grids, SIAM J. Numer. Anal. 41 (2003), no. 6, 2313-2332 (electronic), DOI 10.1137/S0036142901398751. MR2034617 (2004m:65212) 
[7] Peter Binev, Wolfgang Dahmen, and Ron DeVore, Adaptive finite element methods with convergence rates, Numer. Math. 97 (2004), no. 2, 219-268, DOI 10.1007/s00211-003-0492-7. MR2050077 (2005d:65222)

[8] Folkmar A. Bornemann, Bodo Erdmann, and Ralf Kornhuber, A posteriori error estimates for elliptic problems in two and three space dimensions, SIAM J. Numer. Anal. 33 (1996), no. 3, 1188-1204, DOI 10.1137/0733059. MR.1393909(98a:65161)

[9] Willy Dörfler, A convergent adaptive algorithm for Poisson's equation, SIAM J. Numer. Anal. 33 (1996), no. 3, 1106-1124, DOI 10.1137/0733054. MR.1393904 (97e:65139)

[10] Willy Dörfler and Ricardo H. Nochetto, Small data oscillation implies the saturation assumption, Numer. Math. 91 (2002), no. 1, 1-12, DOI 10.1007/s002110100321. MR1896084 (2003e:65195)

[11] Moshe Dubiner, Spectral methods on triangles and other domains, J. Sci. Comput. 6 (1991), no. 4, 345-390, DOI 10.1007/BF01060030. MR1154903 (92k:76061)

[12] Charles F. Dunkl and Yuan Xu, Orthogonal Polynomials of Several Variables, Encyclopedia of Mathematics and its Applications, vol. 81, Cambridge University Press, Cambridge, 2001. MR.1827871 (2002m:33001)

[13] J. S. Hesthaven and T. Warburton, Nodal high-order methods on unstructured grids. I. Timedomain solution of Maxwell's equations, J. Comput. Phys. 181 (2002), no. 1, 186-221, DOI 10.1006/jcph.2002.7118. MR.1925981 (2003f:78034)

[14] George Em Karniadakis and Spencer J. Sherwin, Spectral/hp Element Methods for Computational Fluid Dynamics, 2nd ed., Numerical Mathematics and Scientific Computation, Oxford University Press, New York, 2005. MR2165335 (2006j:65001)

[15] Tom Koornwinder, Two-variable analogues of the classical orthogonal polynomials, Theory and application of special functions (Proc. Advanced Sem., Math. Res. Center, Univ. Wisconsin, Madison, Wis., 1975), Academic Press, New York, 1975, pp. 435-495. Math. Res. Center, Univ. Wisconsin, Publ. No. 35. MR0402146 (53 \#5967)

[16] Khamron Mekchay and Ricardo H. Nochetto, Convergence of adaptive finite element methods for general second order linear elliptic PDEs, SIAM J. Numer. Anal. 43 (2005), no. 5, 18031827 (electronic), DOI 10.1137/04060929X. MR2192319 (2006i:65201)

[17] Joseph Proriol, Sur une famille de polynomes à deux variables orthogonaux dans un triangle (French), C. R. Acad. Sci. Paris 245 (1957), 2459-2461. MR0095994 (20 \#2492)

[18] Sergey Repin, A Posteriori Estimates for Partial Differential Equations, Radon Series on Computational and Applied Mathematics, vol. 4, Walter de Gruyter GmbH \& Co. KG, Berlin, 2008. MR2458008 (2010b:35004)

[19] Rob Stevenson, Optimality of a standard adaptive finite element method, Found. Comput. Math. 7 (2007), no. 2, 245-269, DOI 10.1007/s10208-005-0183-0. MR2324418(2008i:65272)

[20] Gábor Szegő, Orthogonal Polynomials, 4th ed., American Mathematical Society, Providence, R.I., 1975. American Mathematical Society, Colloquium Publications, Vol. XXIII. MR 0372517 (51 \#8724)

[21] Rüdiger Verfürth, A Posteriori Error Estimation Techniques for Finite Element Methods, Numerical Mathematics and Scientific Computation, Oxford University Press, Oxford, 2013. MR 3059294

Korteweg-de Vries Institute for Mathematics, University of Amsterdam, P.O. Box 94248, 1090 GE Amsterdam, Netherlands

E-mail address: T.H.Koornwinder@uva.nl

Institut Für Mathematik, Universität Zürich, Winterthurerstrasse 190, CH-8057 ZüRICH, SwITZERLAND

E-mail address: stas@math.uzh.ch 